\title{
Combining Web Squared and Serious Games for Crossroad Accessibility
}

\author{
Claudio E. Palazzi \\ Dipartimento di Matematica Pura e Applicata \\ Università degli Studi di Padova \\ Padova, Italy \\ e-mail: cpalazzi@math.unipd.it
}

\author{
Gustavo Marfia, Marco Roccetti \\ Dipartimento di Scienze dell' Informazione \\ Università degli Studi di Bologna \\ Bologna, Italy \\ e-mail: $\{$ marfia, roccetti $\} @$ cs.unibo.it
}

\begin{abstract}
The magic trick of the serious game approach lies in the fact that a positive aim can be reached by attracting people in an entertaining task. Yet, the real impact of this technology on our society will happen when it will be utilized to gather the contribution of thousands, maybe millions, of players all around the world to address crucial and unsolved problems. To this aim, we adopt the Web Squared paradigm, combining serious games with crowd-sourcing and automatic data gathering from smartphones' sensors. We show how this combination can create a major force able to tackle serious challenges considered too complex for single users and even for computers. As a proof-of-concept, we discuss a solution able to automatically generate a Google Maps-like service also including information about traffic lights with audible signals. As a final result, our solution improves crossroad accessibility for blind pedestrians by exploiting the latest Web Squared and serious game technology.
\end{abstract}

Keywords-accessibility; blind user; serious game; smartphone; Web Squared

\section{INTRODUCTION}

Games are very popular for their entertainment purpose that attracts people of every age and gender. However, considering games just as a matter of fun and money is definitely wrong. Games also represent a challenging topic for researchers all over the world. One of the reasons is the correlation between problems that emerge in developing innovative game experiences and those typical of other conventional research fields in computer science. We can hence state that games are factually contributing to knowledge advancement.

In this context, serious games are specifically intended to produce benefits for the user or for other people as a collateral effect of playing. In essence, serious games are applications that couple the pure entertainment with a serious purpose [1]. This feature is generally exploited to educate, train, heal, advertise, and inform. DroidGlove is a representative example of serious games designed for the physical rehabilitation of the user [2]. Through accelerator sensors in the patient's smartphones, it verifies whether wrist rehabilitation exercises are accurately performed; results can be sent in real time to a remote physiatrist.

Other interesting examples are games intended to make people aware about the counter-effects in using violence to resolve the problem of terrorism [3]. Any player can decide whether to stay inactive or start bombing terrorists in a certain village where there also many civilians are present. The player will probably try to eliminate some terrorists, but any collateral victim of those bombs creates new terrorists (the victim's familiars and friends), thus making impossible to eliminate all terrorists and actually increasing their number with respect to the beginning of the game.

These few examples show how serious games can be useful for many purposes. Yet, their current use is very limited with respect to their potentiality as does not consider, for instance, the enormous potential offered today by smartphones and their sensors (i.e., accelerometer, GPS, microphone, camera).

The horizons of this context can be pushed further by enabling new interconnecting technologies and creating serious games characterized as pervasive. In the era of Web 2.0, with the approaching Web Squared advent [4], more can be done that can be beneficial for the society as a whole. The players' teamwork could produce results that are more valuable than the sum of those achievable by individuals [5]. Even better, information automatically generated by sensors available on smart phones may integrate the data generated by the community of players, producing new intelligence [6].

As a demonstration of this statement, we have imagined how a pervasive serious game could help users with sight impairments. In particular, we noticed that a very useful Web 2.0 tool such as Google Maps provides routes for cars and pedestrians but with no information about the accessibility of the path.

Instead, it would be interesting to integrate in the route search also information about the presence at crossroads of traffic signals with audible signals. This new functionality requires the existence of a database of information about each traffic light at each crossroads. Unfortunately, this database does not exist and cannot be created by just hiring somebody to verify all crossroads and populate the database: it would be too time consuming and too expensive.

To this aim, our original approach proposes to utilize the pervasive presence of smartphones in our cities and associate it with a game for users. In essence, through a rewarding mechanism, users will be motivated in participating in a serious game that involves the recording of as many audible signals as possible from different traffic lights. All the recorded audio files will be transmitted toward a server hosted by a cloud service where they will be processed and possibly utilized to compose the aforementioned database. 
The rest of this paper is organized as follows. In Section II, we provide background information on the considered scenario highlighting emerging motivations for our work. Section III presents our serious game, the adopted rewarding strategy for players and the general system architecture. Finally, in Section IV we draw conclusion and delineate future developments.

\section{BACKGROUND AND MOTIVATION}

The usefulness of serious games is proven by many available titles. The main idea behind the serious game approach lies in the fact that a positive aim can be reached through attracting people in an entertaining task. Yet, we argue that the positive development process on our society generated by the serious game paradigm is still very slow, with limited outcomes, not considering automatically generated data, and definitely not exploiting all of its potential.

The real impact of serious games on our society will happen when they will be utilized to gather the contribution of thousands, maybe millions, of player all around the world to address crucial and unsolved problems. In essence, we are waiting for serious games to be combined with crowdsourcing and mobile users with sensor-equipped smart phones. This way, we will be able to address serious challenges that are currently considered too complex for single users and even for computers, but could be solved with the help of a multitude of players.

This revolution is already started as demonstrated by popular applications such as Google Image Labeler, which exploits game dynamics between two users to overcome the limitation of computers in tagging images [7]. Google Image Labeler is a web application that leaves this task to users who have to compete in suggesting tags, until the same tag is proposed by both players; then, that tag is associated to the image. Beside users' fun, the purpose of this game is to create a database of images with appropriate tags, overcoming the inability of computers in tagging/recognizing images. Indeed, once integrated with any simple keywords-based search engine, this database allows to simply using textual search keys to retrieve appropriate images. This example shows how serious games can exploit crowd-sourcing to tackle complex issues. Yet, this is not enough.

In fact, even this case is based on the classic game model with static users connected through their PCs; this neglects the enormous potential offered today by the more and more popular smart phones and their sensors (i.e., accelerometer, GPS, microphone, camera).

Instead, as anticipated in Section I, the horizons of this context can be pushed further by merging the paradigms of serious games and Web Squared.

The Web Squared paradigm regards the widespread presence of sensors in the environment due to the popularity of smartphones. Indeed, smartphones are slowly but surely moving the Web from our desks to our pockets. The collective intelligence of the Web is not necessarily forced to come from our keyboards, but it can also be automatically generated by sensors. Indeed, smartphones' sensors reveal where we are, in what direction we are looking at, at what speed we are moving and much more.

The opportunities and information generated by the Web and the widespread participation of users and sensors are growing exponentially thus deserving the term squared for this kind of Web application.

Our aim and original contribution in this paper is to demonstrate how serious games can be coupled with the Web Squared paradigm to solve complex technical issues and improve our society. In particular, our case study regards the distributed gathering of information related to traffic lights with audible signals so as to enable accessible route search for sight impaired users on Google Maps-like services.

We have to mention that solutions have been proposed by researchers to support pedestrians with impairments through specifically designed route generators [8]-[11]. However, these solutions are based on the existence of accessibility information related to each road, whereas, the crucial problem of factually gathering the accessibility-related information remains unsolved. This is the very missing part that our proposed solution is providing.

\section{The Proposed SERIOUS GAME}

To encourage the contribution of as many people as possible, willing to record traffic light audible signals and transmit them toward the server cloud, we devised a serious game where each player receives points for the provided information. Our serious game has to enable the creation of a database with information about the location of traffic lights with audible signals. Main principles of our game are as follows:

- the aim of the game is to collect as many points as possible;

- to gain points, players have to record as many audible signals from different traffic lights as possible;

- all the recorded audio files are associated with geographical coordinates provided by the smartphone's GPS and then transmitted toward remote servers;

- the player receives points depending on the contribution's trustworthiness and originality.

\section{A. Rewarding Strategy}

Each player receives a certain amount of game points each time she/he records the audible signal of a traffic light she/he had not recorded before; local GPS information are used by the smartphone to determine whether that traffic light was already considered by the same user.

One issue that our serious game has to face regards the fact that we cannot be sure that all traffic lights in a town will be considered by players. Traffic lights in crowded parts of the city will gain the interest of a larger number of players. This is acceptable as popular areas in a town are popular even for people with sight impairments. It is hence useful to have those areas quickly included in the database. Yet, after an initial stage, we would like to have as many traffic lights as possible considered by our serious game 
players. To this aim, in our game, when an audible signal is recorded from a position that was not yet present in the database, the player's total score is increased by $10 \%$ thus providing a significant motivation in looking forward for unrecorded data.

Traffic lights that have been recorded in the database are also visible on a Google Maps-like service with, as a further reward, the name (or nickname) of the first person that identified that traffic light as accessible (i.e. endowed with audible signal).

Through a map available on the Web, users can also indicate that a traffic light that has been wrongly identified as accessible. In case several users agree on the fact that a traffic light is not (anymore) accessible then twice the points previously given to players associated with that traffic lights are removed so as to discourage wrong advertising.

Some points are removed even if the same player sends information about the same traffic light more than once.

In summary, this rewarding scheme aims at encouraging players to explore new crossroads and provide new information about their accessibility, rewarding particularly those who first identify a traffic light with audible signal. At the same time, possible cheating behaviours are discouraged through penalities.

\section{B. System Architecture}

The main components of our system architecture are reported in Fig. 1. In essence, players have to install our serious game application on their smartphones. Whenever a player finds a new accessible traffic light, she/he can run our serious game and record the audible signal (Fig. 2). This file is associated with the geographic coordinates automatically gathered through the GPS within the smartphone (in a Web Squared fashion) and then sent to remote servers as soon as connectivity allows Internet communication.
To avoid system bottlenecks and the need for expensive fixed hardware infrastructure, we designed our system to involve cloud computing instead of traditional single servers. Our software server can hence be hosted by a cloud service such as Google's Cloud [12] or Amazon Elastic Compute Cloud [13].

The server hosted by the cloud service is responsible for receiving, processing, and storing all data from players to determine whether a transmitted content is actually an audible signal and corresponds to a geographic location that has not been utilized before by the same player.

Moreover, the server is also responsible to organize data in a specific database to make them available on the Web through a Google Maps-like service. In essence, any user (but probably users having sight impairments) who needs to walk through a certain area can query for an accessible route having traffic lights with audible signals (Fig. 3). By interrogating the database populated thanks to our serious game, the server computes and returns the most suitable route for a person with sight impairments (in a format accessible for people with sight impairment; e.g. audio).

Finally, the server is clearly responsible also to assign, remove and store game points for every player.

\section{CONCLUSION AND Future WORK}

In this paper we have discussed how serious games can be coupled with the Web Squared paradigm to gather the contribution of a multitude of players worldwide and solve crucial technological problems for the development of our society. To this aim, we have presented the design of a system able to automatically generate a Google Maps-like service also including information about traffic lights with audible signals. Our solution effectively improves the digital inclusion of people with impairments.

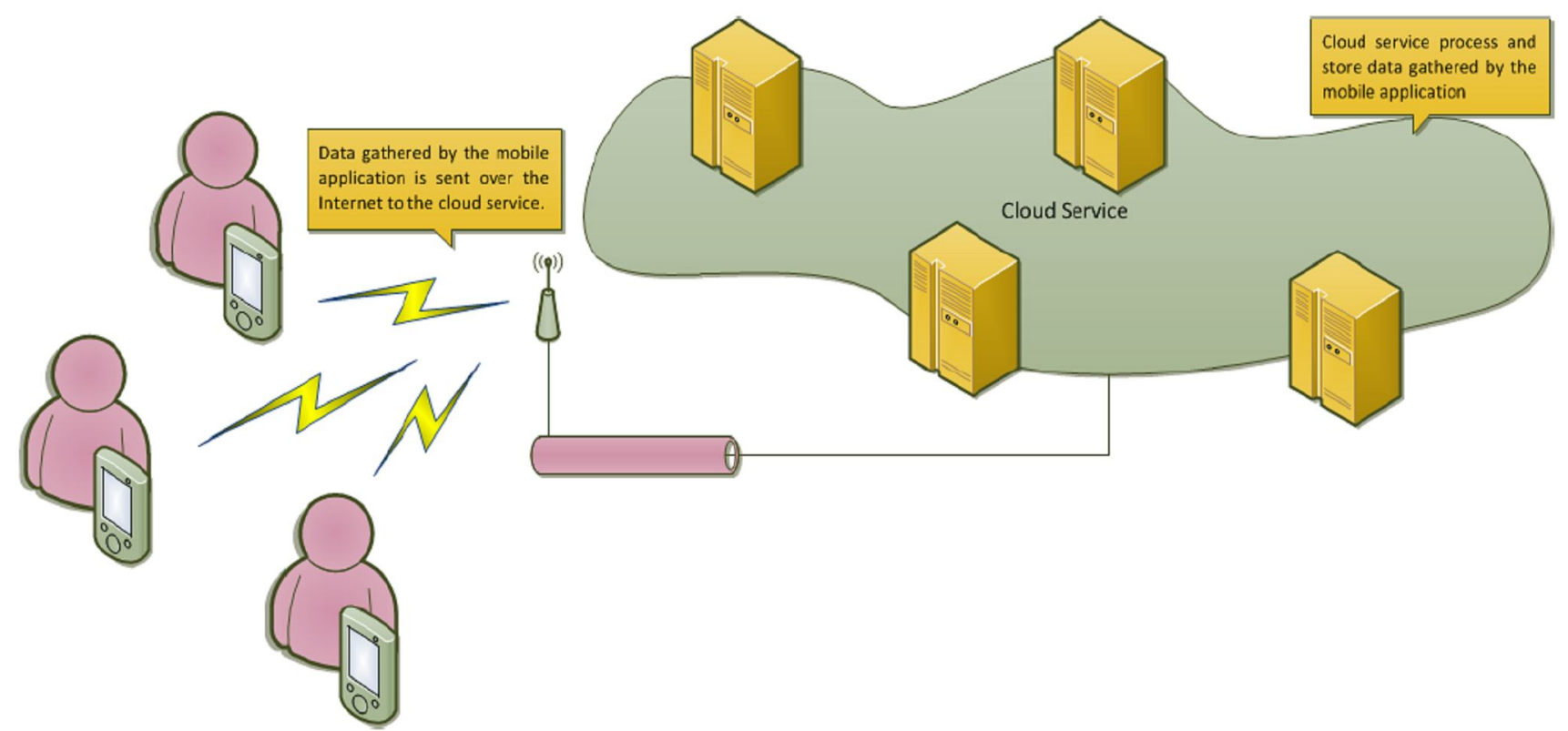

Figure 1. General system architecture. 


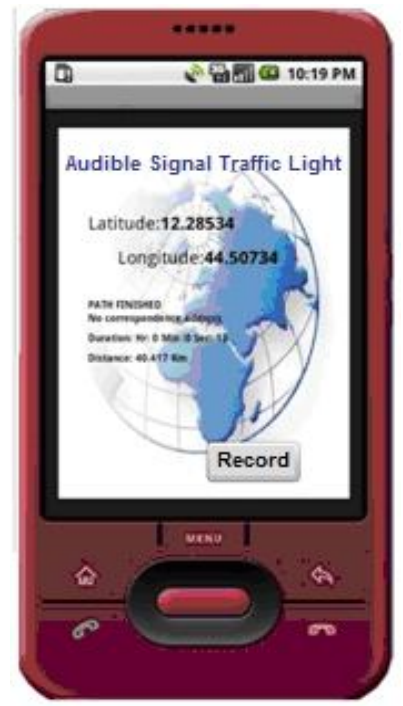

Figure 2. Screenshot example of our serious game.

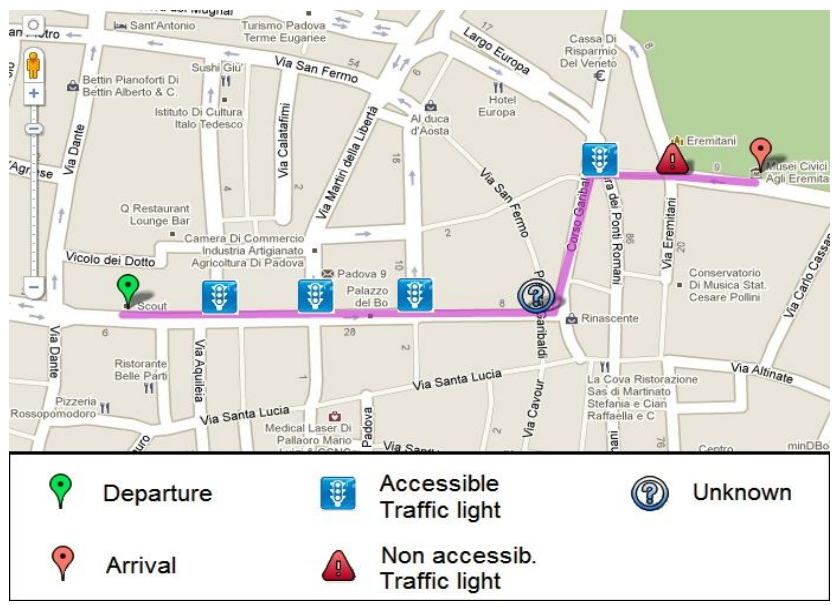

Figure 3. Screenshot example of our Web based application

This work is at a preliminary stage and can be extended in several directions; we cite just a couple among the main ones we are currently investigating. For instance, we need to develop a technical solution to recognize whether a recorded audio file is indeed an audible signal of a traffic light. A viable solution could be to utilize supervised learning approach to this aim [14].

Furthermore, we are also interested in having the whole system working in a Web Squared fashion, with no intervention by humans. In other words, we would like to have smartphones able to autonomously detect their proximity to a traffic light, record, and transmit the audio so as to verify whether there is an audible signal. To identify when a smartphone is close to a traffic light, the GPS location and a map could be exploited. Yet intensive GPS utilization could quickly drain the battery. Therefore, an alternative approach could make use of movement patterns sensed by the accelerometer so as to identify when a user may have stopped at a crossroad and then walked again through it: the latter is the moment when the audio has to be recorded to detect a potential accessible traffic light.

Finally, we would also like to investigate the possibility to manage the whole system in a completely distributed way, with no central server. Inspiration about the possible design and solutions could come from recent wireless sensor network research [15], [16].

\section{ACKNOWLEDGMENT}

Partial financial support for this work is provided by the Università degli Studi di Padova through its research funding program. Moreover, our gratitude goes to Ombretta Gaggi, Alberto Franco and Marco Sbrignadello for the technical discussion about topics related to this work.

\section{REFERENCES}

[1] D. R. Michael, S. L. Chen, Serious Games: Games that Educate, Train and Inform; Muska and Lipman/Premier-Trade Publisher, 2005.

[2] D. Deponti, D. Maggiorini, C. E. Palazzi, "DroidGlove: An AndroidBased Application for Wrist Rehabilitation", Proc. of IEEE International Conference on Ultramodern Telecommunications (ICUMT 2009), St. Petersburg, Russia, Oct 2009.

[3] G. Frasca, Newsgaming - September 12. Available online http://www.newsgaming.com/games/index12.htm

[4] T. O'Reilly, J. BATTELLE, Web Squared: Web 2.0 Five Years on. Available online http://www.web2summit.com/web2009/public/schedule/detail/10194

[5] S. Ferretti, M. Furini, C. E. Palazzi, M. Roccetti, P. Salomoni, "WWW Recycling for a Better World", Communications of the ACM, 53(4):139-143, Apr 2010.

[6] C. E. Palazzi, L. Teodori, M. Roccetti, "PATH 2.0: A Participatory System for the Generation of Accessible Routes", Proc. of IEEE ICME 2010, Singapore, Jul 2010.

[7] Google Image Labeler. Available online http://images.google.com/imagelabeler/

[8] T. Völkel, G. Weber, „RouteChecker: Personalized Multicriteria Routing for Mobility Impaired Pedestrians", Proc. of the 10th ACM ASSETS'08. Halifax, Canada, Oct 2008.

[9] P. Kasemsuppakorn, H. A. Karimi, "Personalised Routing for Wheelchair Navigation", Journal of Location Based Services, 3(1):24-54, 2009.

[10] L. Beale, K. Field, D. Briggs, P. Picton, H. Matthews, "Mapping for Wheelchair Users: Route Navigation in Urban Spaces", The Cartographic Journal, Maney Publishing, 43(1):66-81, Jan 2006.

[11] A. Sobek, H. Miller, "U-Access: A Web-based System for Routing Pedestrians of Differing Abilities", Journal of Geographical Systems, Springer. 8(3):269-287, Sep 2006.

[12] Google Apps for Business - Google's Cloud. Available online http://www.google.com/apps/intl/en/business/cloud.html

[13] Amazon Web Services - Amazon Elastic Compute Cloud (Amazon EC2). Available online http://aws.amazon.com/ec2/

[14] K. Lee, M. Slaney, "Automatic Chord Recognition from Audio Using an HMM with Supervised Learning", Proc. of the 7th International Conf. on Music Information Retrieval, Victoria, Canada, Oct 2006.

[15] N. Aitsaadi, N. Achir, K. Boussetta, G. Pujolle, "A Tabu Search WSN Deployment Method for Monitoring Geographically Irregular Distributed Events", Sensors journal, Special Issue on Wireless Sensor Technologies and Applications. Mar 2009.

[16] N. Aitsaadi, N. Achir, K. Boussetta, G. Pujolle , "Multi-Objectives WSN Deployment: Quality of Monitoring, Connectivity and Lifetime", Proc. of IEEE International Conference on Communications (ICC 2010), Cape Town, South Africa, May 2010. 